\title{
Fixed points of Suzuki type generalized multivalued mappings in fuzzy metric spaces with applications
}

\author{
Naeem Saleem ${ }^{1 *}$, Basit Ali², Mujahid Abbas $^{3}$ and Zahid Raza ${ }^{1}$
}

\section{"Correspondence:}

naeem.saleem2@gmail.com

'Department of Mathematics,

National University of Computer

and Emerging Sciences, Lahore, Pakistan

Full list of author information is available at the end of the article

\begin{abstract}
The aim of this paper is to introduce a class of multivalued mappings satisfying a Suzuki type generalized contractive condition in the framework of fuzzy metric spaces and to present fixed point results for such mappings. Some examples are presented to support the results proved herein. As an application, a common fixed point result for a hybrid pair of single and multivalued mappings is obtained. We show the existence and uniqueness of a common bounded solution of functional equations arising in dynamic programming. Our results generalize and extend various results in the existing literature.
\end{abstract}

MSC: $47 \mathrm{H} 10 ; 47 \mathrm{H} 04 ; 47 \mathrm{H} 07$

Keywords: Suzuki type mapping; fuzzy metric space; fixed point; multivalued mapping; $t$-norm

\section{Introduction and preliminaries}

One of the basic fixed point theorems with a wide range of applications in all types of analyses is the Banach (or Banach-Cassioppoli) contraction principle due to Banach [1]. The Banach contraction principle [1] is a simple and powerful result including iterative methods for solving linear, nonlinear, differential, integral, and difference equations. Due to its applications in mathematics and other related disciplines, this principle has been generalized in many directions. Banach contractions are continuous mappings. Kannan [2] proved fixed point theorems for mappings which are not necessarily continuous. Kikkawa and Suzuki [3] further generalized Banach contractions and Kannan mappings. Suzuki [4] obtained a variant of Banach contraction principle that characterizes metric completeness by using a different type of contractive condition. There are multivalued generalizations of Banach contraction principle (see [5-8]). Đorić and Lazović [9] extended a Suzuki type fixed point theorem and obtained fixed points of a multivalued mapping. They also gave applications of their results in dynamic programming.

On the other hand, the evolution of fuzzy mathematics commenced with an introduction of the notion of fuzzy sets by Zadeh [10] in 1965 as a new way to represent vagueness in everyday life. There are different notions of fuzzy metric spaces. Kramosil and Michalek [11] introduced the notion of fuzzy metric space by using continuous $t$-norms, which generalizes the concept of probabilistic metric space to fuzzy situation. Moreover, George and Veeramani $[12,13]$ modified the concept of fuzzy metric spaces and obtained a Hausdorff

2015 Saleem et al: licensee Springer. This is an Open Access article distributed under the terms of the Creative Commons Attribution License (http://creativecommons.org/licenses/by/4.0), which permits unrestricted use, distribution, and reproduction in any medium, provided the original work is properly credited. 
topology for this kind of fuzzy metric spaces. Recently, Gregori et al. [14] gave applications of fuzzy metrics to color image process and used the concept of fuzzy metric to filter noisy images and in other engineering problems of special interests. Fixed point theory in fuzzy metric spaces has been studied by a number of authors (compare for details [1527]). Rodríguez-López and Romaguera [28] introduced Hausdorff fuzzy metric on a set of nonempty compact subsets of a fuzzy metric space.

In this paper, we obtain Suzuki type fixed point theorems for multivalued mappings in fuzzy metric spaces. Our results extend the comparable results given in $[3,4,7]$ to fuzzy metric spaces.

The following definitions and well-known results will be needed in the sequel.

Definition $1.1([29])$ A binary operation $*:[0,1]^{2} \rightarrow[0,1]$ is called a continuous $t$-norm if

(1) $*$ is associative and commutative;

(2) $*:[0,1]^{2} \rightarrow[0,1]$ is continuous (note that a $t$-norm is continuous if it is continuous as a mapping under usual topology on $\left.[0,1]^{2}\right)$;

(3) $a * 1=a$ for all $a \in[0,1]$;

(4) $a * b \leq c * d$ whenever $a \leq c$ and $b \leq d$.

Some basic examples of continuous $t$-norms are $\wedge, \cdot$ and $*_{L}$, where, for all $a, b \in[0,1]$, $a \wedge b=\min \{a, b\}, a \cdot b=a b$, and $*_{L}$ is the Lukasiewicz $t$-norm defined by $a *_{L} b=\max \{a+$ $b-1,0\}$.

It is easy to check that $*_{L} \leq \cdot \leq \wedge$. In fact $* \leq \wedge$ for all continuous $t$-norm $*$.

Definition $1.2([12,13])$ Let $X$ be a nonempty set and $*$ be a continuous $t$-norm. A fuzzy set $M$ on $X \times X \times(0,+\infty)$ is said to be a fuzzy metric on $X$ if for any $x, y, z \in X$ and $s, t>0$, the following conditions hold:

(i) $M(x, y, t)>0$;

(ii) $x=y$ if and only if $M(x, y, t)=1$ for all $t>0$;

(iii) $M(x, y, t)=M(y, x, t)$;

(iv) $M(x, z, t+s) \geq M(x, y, t) * M(y, z, s)$ for all $t, s>0$;

(v) $M(x, y, \cdot):(0,+\infty) \rightarrow(0,1]$ is continuous.

The triplet $(X, M, *)$ is called a fuzzy metric space. Each fuzzy metric $M$ on $X$ generates Hausdorff topology $\tau_{M}$ on $X$ whose base is the family of open $M$-balls $\left\{B_{M}(x, \varepsilon, t): x \in\right.$ $X, \varepsilon \in(0,1), t>0\}$, where

$$
B_{M}(x, \varepsilon, t)=\{y \in X: M(x, y, t)>1-\varepsilon\} .
$$

Note that a sequence $\left\{x_{n}\right\}$ converges to $x \in X$ (with respect to $\tau_{M}$ ) if and only if $\lim _{n \rightarrow \infty} M\left(x_{n}, x, t\right)=1$ for all $t>0$.

It is well known that for each $x, y \in X, M(x, y, \cdot)$ is a nondecreasing function on $(0,+\infty)$ (see [17]). Moreover, every fuzzy metric space $X$ (in the sense of George and Veeramani [12]) is metrizable, that is, there exists a metric $d$ on $X$ which induces a topology that agrees with $\tau_{M}$ (see [18]). Conversely if $(X, d)$ is a metric space and $M_{d}: X \times X \times(0,+\infty) \rightarrow(0,1]$ is defined as

$$
M_{d}(x, y, t)=\frac{t}{t+d(x, y)}
$$


for all $t>0$, then $\left(X, M_{d}, \wedge\right)$ is a fuzzy metric space called the standard fuzzy metric space induced by the metric $d$ (see [12]). The topologies induced by the standard fuzzy metric and the corresponding metric are the same [19].

A sequence $\left\{x_{n}\right\}$ in a fuzzy metric space $X$ is said to be a Cauchy sequence if for each $\varepsilon \in(0,1)$, there exists $n_{0} \in \mathbb{N}$ such that $M\left(x_{n}, x_{m}, t\right)>1-\varepsilon$ for all $n, m \geq n_{0}$. A fuzzy metric space $X$ is complete [13] if every Cauchy sequence converges in $X$. A subset $A$ of $X$ is closed if for each convergent sequence $\left\{x_{n}\right\}$ in $A$ with $x_{n} \rightarrow x$, we have $x \in A$. A subset $A$ of $X$ is compact if each sequence in $A$ has a convergent subsequence. We denote the set of all nonempty compact subsets of $X$ by $\mathcal{K}(X)$. Let $T: X \rightarrow \mathcal{K}(X)$ be a multivalued mapping and $g$ be a self-mapping on $X$. Further a point $x \in X$ is called: (1) a fixed point of $T$ if and only if $x \in T x$, (2) a coincidence point of $T$ and $g$ if and only if $g x \in T x$, (3) a common fixed point of $T$ and $g$ if and only if $x=g x \in T x$.

We denote the set of all fixed points of $T$ by $F(T)$ and the set of all coincidence points (common fixed points) of mappings $T$ and $g$ by $C(g, T)(F(g, T))$. A mapping $g$ is called $T$-weakly commuting at some point $x \in X$ if $g^{2} x \in \operatorname{Tgx}$. The hybrid pair $(T, g)$ is called $w$-compatible if $g T x \subseteq T g x$ whenever $x \in C(g, T)$ (see [30] and the references therein).

Definition 1.3 ([28]) A fuzzy metric $M$ is said to be continuous on $X^{2} \times(0, \infty)$ if

$$
\lim _{n \rightarrow \infty} M\left(x_{n}, y_{n}, t_{n}\right)=M(x, y, t)
$$

whenever $\left\{\left(x_{n}, y_{n}, t_{n}\right)\right\}$ is a sequence in $X^{2} \times(0, \infty)$ which converges to a point $(x, y, t) \in$ $X^{2} \times(0, \infty)$.

Proposition 1.4 ([28]) Let $(X, M, *)$ be a fuzzy metric space. Then $M$ is a continuous function on $X \times X \times(0, \infty)$.

Consistent with [28], we recall the notion of Hausdorff fuzzy metric induced by a fuzzy metric $M$ as follows: For $x \in X, A \in \mathcal{K}(X)$ and $A, B \in \mathcal{K}(X)$ define

$$
H_{M}(A, B, t)=\min \left\{\inf _{a \in A} M(a, B, t), \inf _{b \in B} M(A, b, t)\right\}
$$

for all $t>0$, where $M(x, A, t)=\sup _{a \in A} M(x, a, t)$. Then $H_{M}$ is called the Hausdorff fuzzy metric induced by the fuzzy metric $M$. The triplet $\left(\mathcal{K}(X), H_{M}, *\right)$ is called Hausdorff fuzzy metric space.

Lemma 1.5 ([28]) Let $X$ be a fuzzy metric space. Then, for each $a \in X, B \in \mathcal{K}(X)$ and $t>0$, there is $b_{0} \in B$ such that $M(a, B, t)=M\left(a, b_{0}, t\right)$.

Lemma 1.6 ([31]) Let $g$ be a self-mapping on a nonempty set $X$. Then there exists a subset $E \subseteq X$ such that $g(E)=g(X)$ and $g: E \rightarrow X$ is one-to-one.

Let $\Omega=\{\eta:[0,1] \rightarrow[0,1], \eta$ is continuous, nondecreasing and $\eta(t)>t$ for $t \in(0,1)\}$.

Note that $\eta(1)=1$ and $\eta(0)=0$, then $\eta(t) \geq t$ for all $t \in[0,1]$.

A sequence $\left\{t_{n}\right\}$ of positive real numbers is said to be $s$-increasing (for further details, see [19]) if there exists $n_{0} \in \mathbb{N}$ such that

$$
t_{m+1} \geq t_{m}+1
$$


for all $m \geq n_{0}$. In a fuzzy metric space $(X, M, \wedge)$, an infinite product (compare [32]) is denoted by

$$
M\left(x, y, t_{1}\right) \wedge M\left(x, y, t_{2}\right) \wedge \cdots \wedge M\left(x, y, t_{n}\right) \wedge \cdots=\prod_{i=1}^{\infty} M\left(x, y, t_{i}\right)
$$

for all $x, y \in X$.

\section{Fixed points of multivalued mappings in fuzzy metric space}

Suppose that

$$
\varphi(r)= \begin{cases}1, & 0 \leq r<\frac{1}{2} \\ 1-r, & \frac{1}{2} \leq r<1\end{cases}
$$

In this section, we obtain several fixed point theorems for multivalued mappings satisfying a Suzuki type contractive condition in the setup of fuzzy metric spaces. We start with the following result.

Theorem 2.1 Let $(X, M, \wedge)$ be a complete fuzzy metric space and $T: X \rightarrow \mathcal{K}(X)$ be a multivalued mapping. Suppose that there exists $r$ in $[0,1)$ such that for each $x, y \in X$ and $\eta \in \Omega$,

$$
M(x, T x, t) \geq M(x, y, \varphi(r) t)
$$

implies

$$
H_{M}(T x, T y, r t) \geq N_{T}^{M}(x, y)
$$

where

$$
N_{T}^{M}(x, y)=\eta(\min \{M(x, y, t), M(x, T x, t), M(y, T y, t), M(x, T y, 2 t), M(y, T x, t)\}) .
$$

Then $F(T) \neq \phi$ provided that the following conditions hold:

(c-1) For each $\varepsilon>0$ and an s-increasing sequence $\left\{t_{n}\right\}$, there exists $n_{0}$ in $\mathbb{N}_{0}$ such that $\prod_{n \geq n_{0}}^{\infty} M\left(x, y, t_{n}\right) \geq 1-\varepsilon$ for all $n \geq n_{0}$.

(c-2) If a sequence $\left\{u_{n}\right\}$ converges to $z$ in $(X, M, \wedge)$, then there exists $n_{1}$ in $\mathbb{N}_{0}$ such that $M\left(u_{n}, x, t\right) \leq M(z, x, t)$ for all $z \neq x$, for all $n \geq n_{1}$ and for all $t>0$.

Proof Let $u_{1}$ be a given point in $X$. Since $T u_{1} \in \mathcal{K}(X)$, we can choose $u_{2} \in T u_{1}$ such that

$$
M\left(u_{2}, T u_{2}, r t\right) \geq H_{M}\left(T u_{1}, T u_{2}, r t\right) .
$$

As $\varphi(r)<1$, so we have

$$
M\left(u_{1}, T u_{1}, t\right) \geq M\left(u_{1}, T u_{1}, \varphi(r) t\right) \geq M\left(u_{1}, u_{2}, \varphi(r) t\right) .
$$


Then, by a given assumption, it follows that

$$
\begin{aligned}
M & \left(u_{2}, T u_{2}, r t\right) \\
& \geq H_{M}\left(T u_{1}, T u_{2}, r t\right) \\
& \geq \eta\left(\min \left\{M\left(u_{1}, u_{2}, t\right), M\left(u_{1}, T u_{1}, t\right), M\left(u_{2}, T u_{2}, t\right), M\left(u_{1}, T u_{2}, 2 t\right), M\left(u_{2}, T u_{1}, t\right)\right\}\right) \\
& \geq \eta\left(\min \left\{M\left(u_{1}, u_{2}, t\right), M\left(u_{1}, u_{2}, t\right), M\left(u_{2}, T u_{2}, t\right), M\left(u_{1}, T u_{2}, 2 t\right), M\left(u_{2}, u_{2}, t\right)\right\}\right) \\
& \geq \eta\left(\min \left\{M\left(u_{1}, u_{2}, t\right), M\left(u_{1}, u_{2}, t\right), M\left(u_{2}, T u_{2}, t\right), M\left(u_{1}, T u_{2}, 2 t\right)\right\}\right) .
\end{aligned}
$$

Since $T u_{2}$ is compact, so by Lemma 1.5 there exists $u_{3} \in T u_{2}$ such that $M\left(u_{2}, T u_{2}, t\right)=$ $M\left(u_{2}, u_{3}, t\right)$. Note that

$$
M\left(u_{1}, T u_{2}, 2 t\right)=M\left(u_{1}, u_{3}, 2 t\right) \geq M\left(u_{1}, u_{2}, t\right) \wedge M\left(u_{2}, u_{3}, t\right) .
$$

Hence we obtain that

$$
M\left(u_{2}, u_{3}, r t\right) \geq \eta\left(\min \left\{M\left(u_{1}, u_{2}, t\right), M\left(u_{2}, u_{3}, t\right)\right\}\right)
$$

If $\min \left\{M\left(u_{1}, u_{2}, t\right), M\left(u_{2}, u_{3}, t\right)\right\}=M\left(u_{2}, u_{3}, t\right)$, then

$$
M\left(u_{2}, u_{3}, r t\right) \geq \eta\left(M\left(u_{2}, u_{3}, t\right)\right)
$$

gives a contradiction. Thus we have

$$
M\left(u_{2}, u_{3}, t\right) \geq \eta\left(M\left(u_{1}, u_{2}, \frac{t}{r}\right)\right)
$$

Continuing this way, we can obtain a sequence $\left\{u_{n}\right\}$ in $X$ such that $u_{n+1} \in T u_{n}$, which satisfies

$$
\begin{aligned}
M\left(u_{n}, u_{n+1}, t\right) & \geq M\left(u_{n-1}, u_{n}, \frac{t}{r}\right) \\
& \geq M\left(u_{n-2}, u_{n-1}, \frac{t}{r^{2}}\right) \geq \cdots \geq M\left(u_{0}, u_{1}, \frac{t}{r^{n}}\right) .
\end{aligned}
$$

Let $t>0, \varepsilon>0, m, n \in \mathbb{N}$ such that $m>n$ and $h_{i}=\frac{1}{i(i+1)}$ for $i \in\{n, n+1, \ldots m-1\}$. As $h_{n}+$ $h_{n+1}+\cdots+h_{m-1}<1$, so we have

$$
\begin{aligned}
M & \left(u_{n}, u_{m}, t\right) \\
& \geq M\left(u_{n}, u_{m},\left(h_{n}+h_{n+1}+\cdots+h_{m-1}\right) t\right) \\
& \geq M\left(u_{n}, u_{n+1}, h_{n} t\right) \wedge M\left(u_{n+1}, u_{n+2}, h_{n+1} t\right) \wedge \cdots \wedge M\left(u_{m-1}, u_{m}, h_{m-1} t\right) \\
& \geq M\left(u_{0}, u_{1}, \frac{h_{n}}{r^{n}} t\right) \wedge M\left(u_{0}, u_{1}, \frac{h_{n+1}}{r^{n+1}} t\right) \wedge \cdots \wedge M\left(u_{0}, u_{1}, \frac{h_{m-1}}{r^{m-1}} t\right) \\
& =M\left(u_{0}, u_{1}, \frac{1}{n(n+1) r^{n}} t\right) \wedge M\left(u_{0}, u_{1}, \frac{1}{(n+1)(n+2) r^{n+1}} t\right) \wedge \cdots
\end{aligned}
$$




$$
\begin{gathered}
\wedge M\left(u_{0}, u_{1}, \frac{1}{m(m-1) r^{m-1}} t\right) \\
\geq \prod_{n=1}^{\infty} M\left(u_{0}, u_{1}, \frac{t}{n(n+1) r^{n}}\right)=\prod_{n=1}^{\infty} M\left(u_{0}, u_{1}, t_{n}\right),
\end{gathered}
$$

where $t_{n}=\frac{t}{n(n+1) r^{n}}$. Since $\lim _{n \rightarrow \infty}\left(t_{n+1}-t_{n}\right)=\infty$, therefore $\left\{t_{n}\right\}$ is an $s$-increasing sequence. Consequently, there exists $n_{0} \in \mathbb{N}$ such that for each $\varepsilon>0$, we have $\prod_{n=1}^{\infty} M\left(u_{0}, u_{1}, t_{n}\right) \geq$ $1-\varepsilon$ for all $n \geq n_{0}$ and hence we obtain $M\left(u_{n}, u_{m}, t\right) \geq 1-\varepsilon$ for all $n, m \geq n_{0}$. Thus $\left\{u_{n}\right\}$ is a Cauchy sequence in $X$. As $X$ is a complete, so there exists a point $z$ in $X$ such that $\lim _{n \rightarrow \infty} M\left(u_{n}, z, t\right)=1$. Now we show that

$$
M(z, T x, r t) \geq \eta(\min \{M(z, x, t), M(x, T x, t)\})
$$

for all $x$ in $X \backslash\{z\}$. Since $\lim _{n \rightarrow \infty} u_{n}=z$, so there exists a positive integer $n_{1} \in N$ such that for all $n \geq n_{1}$, we have

$$
M\left(z, u_{n}, \frac{t}{2}\right) \geq M(z, x, t)
$$

and by (c-2) there exists $n_{2}$ in $\mathbb{N}_{0}$ such that $M\left(u_{n}, x, t\right) \leq M(z, x, t)$ for all $z \neq x, n \geq n_{2}$ and for all $t>0$. Now if we take $n_{3}=\max \left\{n_{1}, n_{2}\right\}$, then we obtain that

$$
\begin{aligned}
M\left(u_{n}, T u_{n}, t\right) & \geq M\left(u_{n}, z, \frac{t}{2}\right) \wedge M\left(z, T u_{n}, \frac{t}{2}\right) \geq M\left(u_{n}, z, \frac{t}{2}\right) \wedge M\left(z, u_{n+1}, \frac{t}{2}\right) \\
& \geq M(z, x, t) \wedge M(z, x, t)=M(z, x, t) \geq M\left(u_{n}, x, t\right) \\
& \geq M\left(u_{n}, x, \varphi(r) t\right)
\end{aligned}
$$

for all $n \geq n_{3}$. Thus, we have

$$
\begin{aligned}
M & \left(u_{n+1}, T x, r t\right) \\
& \geq H_{M}\left(T u_{n}, T x, r t\right) \\
& \geq \eta\left(\min \left\{M\left(u_{n}, x, t\right), M\left(u_{n}, T u_{n}, t\right), M(x, T x, t), M\left(u_{n}, T x, 2 t\right), M\left(x, T u_{n}, t\right)\right\}\right) \\
& \geq \eta\left(\min \left\{M\left(u_{n}, x, t\right), M\left(u_{n}, u_{n+1}, t\right), M(x, T x, t), M\left(u_{n}, T x, 2 t\right), M\left(x, u_{n+1}, t\right)\right\}\right),
\end{aligned}
$$

which on taking limit as $n \rightarrow \infty$ gives

$$
\begin{aligned}
M(z, T x, r t) & \geq \eta(\min \{M(z, x, t), M(x, T x, t), M(z, T x, t), M(x, z, t)\}) \\
& \geq \eta(\min \{M(z, x, t), M(x, T x, t), M(z, T x, 2 t)\}) \\
& \geq \eta(\min \{M(z, x, t), M(x, T x, t),(M(z, x, t) \wedge M(x, T x, t))\}) \\
& \geq \eta(\min \{M(z, x, t), M(x, T x, t)\}) .
\end{aligned}
$$

Hence

$$
M(z, T x, r t) \geq \eta(\min \{M(z, x, t), M(x, T x, t)\})
$$

Now, we show that $z \in T z$. For this, we consider the following cases: 
(a) Take $0 \leq r<\frac{1}{2}$. Assume on the contrary that $z \notin T z$. Let $a \in T z$. Then $a \neq z$ and it follows that

$$
\begin{aligned}
M(a, z, t) & \geq M(a, T z,(1-2 r) t) \wedge M(z, T z, 2 r t) \\
& \geq M(a, a,(1-2 r) t) \wedge M(z, T z, 2 r t) \\
& =M(z, T z, 2 r t) .
\end{aligned}
$$

Using (1) we have

$$
M(z, T a, r t) \geq \eta(\min \{M(z, a, t), M(a, T a, t)\}) .
$$

Also,

$$
M(z, T z, t) \geq M(z, T z, \varphi(r) t) \geq M(z, a, \varphi(r) t) .
$$

Thus, we have

$$
\begin{aligned}
H_{M}(T z, T a, r t) & \geq \eta(\min \{M(z, a, t), M(z, T z, t), M(a, T a, t), M(z, T a, 2 t), M(a, T z, t)\}) \\
& \geq \eta(\min \{M(z, a, t), M(a, T a, t), M(z, T a, 2 t)\}) \\
& \geq \eta(\min \{M(z, a, t), M(a, T a, t),(M(z, a, t) \wedge M(a, T a, t))\}) \\
& \geq \eta(\min \{M(z, a, t), M(a, T a, t)\}) .
\end{aligned}
$$

Now $M(a, T a, t) \geq H_{M}(T z, T a, t)$ implies that

$$
H_{M}(T z, T a, r t) \geq \eta(M(z, a, t))>M(z, a, t) .
$$

Hence

$$
M(z, T a, r t)>M(z, a, t) .
$$

Since $\eta(t)>t$ for $t \in(0,1)$, so for $a \neq z$ we have

$$
\begin{aligned}
M(z, T z, 2 r t) & \geq M(z, a, 2 r t) \geq M(z, T a, r t) \wedge M(T a, a, r t) \\
& \geq M(z, T a, r t) \wedge H_{M}(T a, T z, r t) \\
& >M(z, a, t) \wedge \eta(\min \{M(z, a, t), M(a, T a, t)\}) \\
& \geq M(z, a, t) \wedge \eta(M(z, a, t)) \\
& >M(z, a, t) \wedge M(z, a, t) \\
& =M(z, a, t) \geq M(z, T z, 2 r t) .
\end{aligned}
$$

That is, $M(z, T z, 2 r t)>M(z, T z, 2 r t)$, a contradiction. Consequently $z \in T z$.

(b) Take $\frac{1}{2} \leq r<1$. We claim that

$$
\begin{aligned}
& H_{M}(T x, T z, r t) \\
& \quad \geq \eta(\min \{M(x, z, t), M(x, T x, t), M(z, T z, t), M(x, T z, 2 t), M(z, T x, t)\})
\end{aligned}
$$


for all $x$. If $x=z$, then the above inequality is immediate. If $x \neq z$, then we have

$$
\begin{aligned}
M(x, T x, t) & \geq M(x, z,(1-r) t) \wedge M(z, T x, r t) \\
& \geq M(x, z, \varphi(r) t) \wedge M(z, T x, r t) .
\end{aligned}
$$

Using (2) we have

$$
\begin{aligned}
M(x, T x, t) & \geq M(x, z, \varphi(r) t) \wedge \eta(\min \{M(z, x, t), M(x, T x, t)\}) \\
& \geq M(x, z, \varphi(r) t) \wedge \eta(\min \{M(z, x, t), M(x, T x, t)\}) .
\end{aligned}
$$

If $\min \{M(z, x, t), M(x, T x, t)\}=M(z, x, t)$, then

$$
\begin{aligned}
M(x, T x, t) & \geq M(x, z, \varphi(r) t) \wedge \eta(\min \{M(z, x, t), M(x, T x, t)\}) \\
& \geq M(x, z, \varphi(r) t) \wedge \eta(M(z, x, t)) \\
& \geq M(x, z, \varphi(r) t) \wedge M(z, x, t)=M(x, z, \varphi(r) t) .
\end{aligned}
$$

If $\min \{M(z, x, t), M(x, T x, t)\}=M(x, T x, t)$, then

$$
\begin{aligned}
M(x, T x, t) & \geq M(x, z, \varphi(r) t) \wedge \eta(\min \{M(z, x, t), M(x, T x, t)\}) \\
& \geq M(x, z, \varphi(r) t) \wedge \eta(M(x, T x, t)) .
\end{aligned}
$$

If $M(x, z, \varphi(r) t) \wedge \eta(M(x, T x, t))=M(x, z, \varphi(r) t)$, then we obtain

$$
M(x, T x, t) \geq M(x, z, \varphi(r) t) .
$$

If not, then $M(x, z, \varphi(r) t) \wedge \eta(M(x, T x, t))=\eta(M(x, T x, t))$ gives

$$
M(x, T x, t) \geq \eta(M(x, T x, t))>M(x, T x, t),
$$

a contradiction. Hence

$$
M(x, T x, t) \geq M(x, z, \varphi(r) t) .
$$

Thus the claim follows. Using $x=u_{n}$ in (3) we obtain that

$$
\begin{aligned}
& M(z, T z, t) \\
& \quad=\lim _{n \rightarrow \infty} M\left(u_{n+1}, T z, t\right) \geq \lim _{n \rightarrow \infty} H_{M}\left(T u_{n}, T z, t\right) \\
& \quad \geq \lim _{n \rightarrow \infty} \eta\left(\min \left\{M\left(u_{n}, z, t\right), M\left(u_{n}, T u_{n}, t\right), M(z, T z, t), M\left(u_{n}, T z, 2 t\right), M\left(z, T u_{n}, t\right)\right\}\right) \\
& \quad \geq \lim _{n \rightarrow \infty} \eta\left(\min \left\{M\left(u_{n}, z, t\right), M\left(u_{n}, u_{n+1}, t\right), M(z, T z, t), M\left(u_{n}, T z, 2 t\right), M\left(z, T u_{n}, t\right)\right\}\right) \\
& \quad \geq \eta(M(z, T z, t)) .
\end{aligned}
$$

Since $\eta(t) \geq t$. Hence $\eta(M(z, T z, t))=M(z, T z, t)$ shows that $M(z, T z, t)=1$. Since $T z$ is compact, consequently $z \in T z$. 
Problem 2.2 Does Theorem 2.1 hold for an arbitrary continuous $t$-norm?

Now we present an example of fuzzy metric space that satisfies all the conditions of Theorem 2.1.

Example 2.3 Let $X=\{0,1,2\}$ and $d: X \times X \rightarrow R$ be a metric defined by

$$
\begin{aligned}
& d(0,1)=d(1,0)=2, \quad d(1,2)=d(2,1)=1.5, \\
& d(0,2)=d(2,0)=1, \quad d(0,0)=d(1,1)=d(2,2)=0 .
\end{aligned}
$$

The fuzzy metric $M$ on $X$ is given by

$$
M(x, y, t)=\frac{t^{2}}{t^{2}+d(x, y)} .
$$

Define $T: X \times X \rightarrow C B(X)$ as

$$
T(x)= \begin{cases}\{0\} & \text { if } x=1 \\ \{0,2\} & \text { if } x \neq 1\end{cases}
$$

Let $r=\sqrt{\frac{3}{4}}$ and $\eta=t \frac{9}{10} \in \Omega$. Note that for all $x, y \in X$, except at $x=1=y$,

$$
M(x, T x, t) \geq M(x, y, \varphi(r) t)
$$

holds true. It is sufficient to check the validity of

$$
H_{M}(T x, T y, r t) \geq N_{T}^{M}(x, y)
$$

for all $x, y$ for which (4) holds true. For this, we consider the following cases:

(i) If $x=y=0$, then we have

$$
H_{M}\left(T 0, T 0, \sqrt{\frac{3}{4}} t\right)=1
$$

So (5) holds in this case.

(ii) If $x=0, y=1$ or $x=1, y=0$, then

$$
\begin{aligned}
N_{T}^{M}(x, y) & =\eta(\min \{M(0,1, t), M(0, T 0, t), M(1, T 1, t), M(0, T 1,2 t), M(1, T 0, t)\}) \\
& =\eta(\min \{M(0,1, t), M(0,\{0,2\}, t), M(1,\{0\}, t), M(0,\{0\}, 2 t), M(1,\{0,2\}, t)\}) \\
& =\eta\left(\min \left\{\frac{t^{2}}{t^{2}+d(0,1)}, \frac{t^{2}}{t^{2}+d(0,0)}, \frac{t^{2}}{t^{2}+d(1,0)}, \frac{4 t^{2}}{4 t^{2}+d(0,0)}, \frac{t^{2}}{t^{2}+d(1,2)}\right\}\right) \\
& =\eta\left(\min \left\{\frac{t^{2}}{t^{2}+2}, 1, \frac{t^{2}}{t^{2}+2}, 1, \frac{t^{2}}{t^{2}+1.5}\right\}\right) \\
& =\eta\left(\frac{t^{2}}{t^{2}+2}\right)
\end{aligned}
$$




$$
\begin{aligned}
& H_{M}\left(T 0, T 1, \frac{3}{4} t\right) \\
& =\min \left\{\inf _{x \in T 0} M\left(x, T 1, \sqrt{\frac{3}{4}} t\right), \inf _{y \in T 1} M\left(T 0, T 1, \sqrt{\frac{3}{4}} t\right)\right\} \\
& =\min \left\{\inf _{x \in T 0} M\left(x,\{0\}, \sqrt{\frac{3}{4}} t\right), M\left(\{0,2\}, 0, \sqrt{\frac{3}{4}} t\right)\right\} \\
& =\min \left\{\inf \left\{M\left(0,0, \sqrt{\frac{3}{4}} t\right), M\left(2,0, \sqrt{\frac{3}{4}} t\right)\right\}, M\left(0,0, \sqrt{\frac{3}{4}} t\right)\right\} \\
& =\min \left\{\inf \left\{1, \frac{\frac{3}{4} t^{2}}{\frac{3}{4} t^{2}+1}\right\}, 1\right\}=\frac{\frac{3}{4} t^{2}}{\frac{3}{4} t^{2}+1} .
\end{aligned}
$$

Note that

$$
\frac{\frac{3}{4} t^{2}}{\frac{3}{4} t^{2}+1} \geq\left(\frac{t^{2}}{t^{2}+2}\right)^{\frac{9}{10}}>\frac{t^{2}}{t^{2}+2} .
$$

(iii) If $x=0, y=2$ or $x=2, y=0$, then $H_{M}\left(T 0, T 2, \sqrt{\frac{3}{4}} t\right)=1$ and $N_{T}^{M}(x, y)=\eta\left(\frac{t^{2}}{t^{2}+1}\right)$.

(iv) If $x=1, y=2$ or $x=2, y=1$, then

$$
\begin{aligned}
& N_{T}^{M}(x, y)=\eta(\min \{M(1,2, t), M(1, T 1, t), M(2, T 2, t), M(1, T 2,2 t), M(2, T 1, t)\}) \\
& =\eta(\min \{M(1,2, t), M(1,\{0\}, t), M(2,\{0,2\}, t), M(1,\{0,2\}, 2 t), M(2,\{0\}, t)\}) \\
& =\eta\left(\min \left\{\frac{t^{2}}{t^{2}+d(1,2)}, \frac{t^{2}}{t^{2}+d(1,0)}, \frac{t^{2}}{t^{2}+d(2,2)}, \frac{4 t^{2}}{4 t^{2}+d(1,2)}, \frac{t^{2}}{t^{2}+d(2,0)}\right\}\right) \\
& =\eta\left(\min \left\{\frac{t^{2}}{t^{2}+1.5}, \frac{t^{2}}{t^{2}+2}, 1, \frac{4 t^{2}}{4 t^{2}+1.5}, \frac{t^{2}}{t^{2}+1}\right\}\right) \\
& =\eta\left(\frac{t^{2}}{t^{2}+2}\right) \text {, } \\
& H_{M}\left(T 1, T 2, \sqrt{\frac{3}{4}} t\right) \\
& =\min \left\{\inf _{x \in T 1} M\left(x, T 2, \sqrt{\frac{3}{4}} t\right), \inf _{y \in T 2} M\left(T 1, y, \sqrt{\frac{3}{4}} t\right)\right\} \\
& =\min \left\{\inf _{x \in T 1} M\left(x,\{0,2\}, \sqrt{\frac{3}{4}} t\right), \inf _{y \in T 2} M\left(\{0\}, y, \sqrt{\frac{3}{4}} t\right)\right\} \\
& =\min \left\{M\left(0,\{0,2\}, \sqrt{\frac{3}{4}} t\right), \inf _{y \in T 2}\left\{M\left(0,0, \sqrt{\frac{3}{4}} t\right), M\left(0,2, \sqrt{\frac{3}{4}} t\right)\right\}\right\} \\
& =\min \left\{1, \inf \left\{1, \frac{\frac{3}{4} t^{2}}{\frac{3}{4} t^{2}+1}\right\}\right\}=\frac{\frac{3}{4} t^{2}}{\frac{3}{4} t^{2}+1} \text {. }
\end{aligned}
$$

Note that

$$
\frac{\frac{3}{4} t^{2}}{\frac{3}{4} t^{2}+1} \geq\left(\frac{t^{2}}{t^{2}+2}\right)^{\frac{9}{10}}>\frac{t^{2}}{t^{2}+2}
$$


Hence, for all $x, y \in X$,

$$
H_{M}\left(T x, T y, \frac{3}{4} t\right) \geq \eta\left(N_{T}^{M}(x, y)\right)>N_{T}^{M}(x, y) .
$$

Moreover, for any $s$-increasing sequence $\left\{t_{n}\right\}, \prod_{n=1}^{\infty} M\left(x, y, t_{n}\right)$ is convergent (see [19], Examples 4.10 and 4.11), that is, (c-1) is satisfied. Since only convergent sequences in $X$ are constant sequences $\left\{u_{n}\right\}$, where $u_{n}=c$ and $c \in X$, therefore (c-2) holds true. Thus all the conditions of Theorem 2.1 are satisfied.

Remark 2.4 If we consider the standard fuzzy metric $M_{d}(x, y, t)=\frac{t}{t+d(x, y)}$ in the example above, then all the conditions in Theorem 2.1 are satisfied except (c-1). Indeed, for any $s$-increasing sequence $\left\{t_{n}\right\}$ with $t_{n}=n, \prod_{n=1}^{\infty} M_{d}\left(x, y, t_{n}\right)$ converges to 0 (see [19], Example 4.11).

Remark 2.5 Let $(X, M, \wedge)$ be a complete fuzzy metric space and $T: X \rightarrow \mathcal{K}(X)$ be a multivalued mapping. We modify condition (c-1) in Theorem 2.1 as follows:

(c-3) For each $\varepsilon>0$ and an $s$-increasing sequence $\left\{t_{n}\right\}$, there exist $n_{0} \in \mathbb{N}, u_{0} \in X$ and $u_{1} \in T u_{0}$ such that $\prod_{n=1}^{\infty} M\left(u_{0}, u_{1}, t_{n}\right) \geq 1-\varepsilon$.

If we replace condition (c-3) with (c-1) in Theorem 2.1 and start the proof of the theorem with initial points $u_{0} \in X$ and $u_{1} \in T u_{0}$ for which (c-3) holds, then the conclusion of Theorem 2.1 remains the same.

Example 2.6 Let $X=\mathbb{N}$ and $P: X \times X \times(0, \infty) \rightarrow(0,1]$ be a fuzzy metric defined by

$$
P(x, y, t)= \begin{cases}1 & \text { if } x=y \\ \frac{1}{2} & \text { if } x \neq y .\end{cases}
$$

Note that $(X, P, \wedge)$ is a complete fuzzy metric space which induces the discrete topology on $X$ and there does not exist any metric $d$ on $X$ satisfying

$$
P(x, y, t)=\frac{t}{t+d(x, y)} .
$$

Moreover, for an $s$-increasing sequence $\left\{t_{n}=n\right\}, \prod_{n=1}^{\infty} P\left(x, y, t_{n}\right)$ converge to 0 (see [19], Examples 4.10 and 4.11). Hence $P$ does not satisfy condition (c-1) of Theorem 2.1. Define $T: X \rightarrow C B(X)$ as

$$
T(x)= \begin{cases}\{1\} & \text { if } x=2 \\ \{1,3\} & \text { if } x \neq 2\end{cases}
$$

If $u_{0}=1$ and $u_{1}=1 \in T u_{0}$, then for any $s$-increasing sequence $\left\{t_{n}\right\}, \prod_{n=1}^{\infty} M\left(u_{0}, u_{1}, t_{n}\right)$ converges to 1 . That is, $P$ satisfies condition (c-3) in Remark 2.5. Note that for any $r \in[0,1)$ and $x \neq y$,

$$
P(x, T x, t) \geq \frac{1}{2}=P(x, y, \varphi(r) t)
$$

is satisfied, whereas

$$
H_{P}(T x, T y, r t) \geq \frac{1}{2}=N_{T}^{P}(x, y)
$$


holds true for all $x, y$ in $X$. Moreover, conditions (c-2) and (c-3) are satisfied. Hence all the conditions of the variant of Theorem 2.1 are satisfied in the setup of $(X, P, \wedge)$.

Corollary 2.7 Let $(X, M, \wedge)$ be a complete fuzzy metric space and $T: X \rightarrow \mathcal{K}(X)$ be a multivalued mapping. Suppose that there exists $r$ in $[0,1)$ such that for each $x, y \in X$ and $\eta \in \Omega$,

$$
M(x, T x, t) \geq M(x, y, \varphi(r) t)
$$

implies

$$
H_{M}(T x, T y, r t) \geq \eta(M(x, y, t)) .
$$

Then there exists a point $z \in X$ such that $z \in$ Tz provided that conditions (c-1) and (c-2) in Theorem 2.1 hold.

Corollary 2.8 Let $(X, M, \wedge)$ be a complete fuzzy metric space and $T: X \rightarrow \mathcal{K}(X)$ be a multivalued mapping. Suppose that there exists $r$ in $[0,1)$ such that for each $x, y \in X$ and $\eta \in \Omega$,

$$
M(x, T x, t) \geq M(x, y, \varphi(r) t)
$$

implies

$$
H_{M}(T x, T y, r t) \geq \eta(\min \{M(x, y, t), M(x, T x, t), M(y, T y, t)\}) .
$$

Then there exists a point $z \in X$ such that $z \in$ Tz provided that conditions (c-1) and (c-2) in Theorem 2.1 hold.

Corollary 2.9 Let $(X, M, \wedge)$ be a complete fuzzy metric space and $T: X \rightarrow \mathcal{K}(X)$ be a multivalued mapping. Suppose that there exists $r$ in $[0,1)$ such that for each $x, y \in X$ and $\eta \in \Omega$,

$$
M(x, T x, t) \geq M(x, y, \varphi(r) t)
$$

implies

$$
H_{M}(T x, T y, r t) \geq \eta(\min \{M(x, y, t), M(x, T x, t), M(y, T x, t)\})
$$

Then there exists a point $z \in X$ such that $z \in$ Tz provided that conditions (c-1) and (c-2) in Theorem 2.1 hold.

Corollary 2.10 Let $(X, M, \wedge)$ be a complete fuzzy metric space and $T: X \rightarrow \mathcal{K}(X)$ be a multivalued mapping. Suppose that there exists $r$ in $[0,1)$ such that for each $x, y \in X$ and $\eta \in \Omega$,

$$
M(x, T x, t) \geq M(x, y, \varphi(r) t)
$$

implies

$$
H_{M}(T x, T y, r t) \geq \eta\left(\left\{\alpha_{1} M(x, y, t)+\alpha_{2} M(x, T x, t)+\alpha_{3} M(y, T y, t)\right\}\right),
$$


where $\sum_{i=1}^{3} \alpha_{i}=1$ and $\eta \in \Omega$. Then there exists a point $z \in X$ such that $z \in$ Tz provided that conditions (c-1) and (c-2) in Theorem 2.1 hold.

Now, in the next theorem, we prove the existence of coincidence and common fixed points of a hybrid pair of single and multivalued mappings.

Theorem 2.11 Let $(X, M, \wedge)$ be a complete fuzzy metric space, $T: X \rightarrow \mathcal{K}(X)$ and $g$ be a self-mapping on $X$ such that $T(X) \subseteq g(X)$. Suppose that there exists $r$ in $[0,1)$ such that for each $x, y \in X, t>0$ and $\eta \in \Omega$,

$$
M(g x, T x, t) \geq M(g x, g y, \varphi(r) t)
$$

implies

$$
H_{M}(T x, T y, r t) \geq \eta(\min \{M(g x, g y, t), M(g x, T x, t), M(g y, T x, t)\}) .
$$

Then $C(g, T) \neq \phi$ provided that the following conditions hold:

(c-4) For each $\varepsilon>0$ and an s-increasing sequence $\left\{t_{n}\right\}$, there exists $n_{0}$ in $\mathbb{N}_{0}$ such that $\prod_{n \geq n_{0}}^{\infty} M\left(g x, g y, t_{n}\right) \geq 1-\varepsilon$.

(c-5) If a sequence $\left\{g u_{n}\right\}$ converges to $g z$ in $X$, then $M\left(g u_{n}, g x, t\right) \leq M(g z, g x, t)$ for all $z \neq x$.

Further, if one of the following conditions holds:

(a) $T$ and $g$ are $w$-compatible, $\lim _{n \rightarrow \infty} g^{n} x=y$ for some $x \in C(g, T), y \in X$ and $g$ is continuous at $y$.

(b) $g$ is $T$-weakly commuting for some $x \in C(g, T)$ and $g x$ is a fixed point of $g$, that is, $g^{2} x=g x$.

(c) $g$ is continuous at $x$ for some $x \in C(g, T)$ and for some $y \in X, \lim _{n \rightarrow \infty} g^{n} y=x$.

Then $F(g, T) \neq \phi$.

Proof By Lemma 1.6, there exists $E \subseteq X$ such that $g: X \rightarrow X$ is one-to-one and $g(E)=g(X)$. We define $A: g(E) \rightarrow K(X)$ by

$$
A(g x)=T(x) \quad \text { for } g x \in g(E) .
$$

As $g$ is one-to-one on $E$, so $A$ is well defined. Further

$$
M(g x, A(g x), t)=M(g x, T x, t) \geq M(g x, g y, \varphi(r) t)
$$

implies

$$
\begin{aligned}
H_{M}(A(g x), A(g y), r t) & =H_{M}(T x, T y, r t) \\
& \geq \eta(\min \{M(g x, g y, t), M(g x, A(g x), t), M(g y, A(g x), t)\}) .
\end{aligned}
$$

Thus, $A$ satisfies (6) and all the conditions of Corollary 2.9. It follows that $A$ has a fixed point $u \in g(E)$. Now we show that $T$ and $g$ have a coincidence point. Since $A$ has a fixed 
point $u$ in $g(E)$ and $T(X) \subseteq g(X)$, so there exists $v \in X$ such that $g v=u$. Hence

$$
u=g v \in A(u)=A(g v)=T(v) .
$$

That is, $v \in X$ is a coincidence point of $T$ and $g$. Hence $C(g, T)$ is nonempty. If (a) holds, then, for some $x \in C(g, T), \lim _{n \rightarrow \infty} g^{n} x=y$, where $y \in X$. Since $g$ is continuous at $y$, so $y$ is a fixed point of $g$. As $T$ and $g$ are $w$-compatible, so $g^{n} x \in C(g, T)$ for all $n \geq 1$. That is, for all $n \geq 1, g^{n} x \in T\left(g^{n-1} x\right)$. Now

$M(g y, T y, t)$

$$
\begin{aligned}
\geq & M\left(g y, g^{n} x, \frac{t}{2}\right) \wedge M\left(g^{n} x, T y, \frac{t}{2}\right) \\
\geq & M\left(g y, g^{n} x, \frac{t}{2}\right) \wedge H_{M}\left(T\left(g^{n-1} x\right), T y, \frac{t}{2}\right) \\
\geq & M\left(g y, g^{n} x, \frac{t}{2}\right) \wedge H_{M}\left(T\left(g^{n-1} x\right), T y, \frac{t}{2}\right) \\
\geq & M\left(g y, g^{n} x, \frac{t}{2}\right) \\
& \wedge \eta\left(\left\{M\left(g^{n} x, g y, \frac{t}{2}\right), M\left(g^{n} x, T\left(g^{n-1} x\right), \frac{t}{2}\right), M\left(g y, T\left(g^{n-1} x\right), \frac{t}{2}\right)\right\}\right) \\
\geq & M\left(g y, g^{n} x, \frac{t}{2}\right) \wedge \eta\left(\left\{M\left(g^{n} x, g y, \frac{t}{2}\right), M\left(g^{n} x, g^{n} x, \frac{t}{2}\right), M\left(g y, g^{n} x, \frac{t}{2}\right)\right\}\right) \\
\geq & M\left(g y, g^{n} x, \frac{t}{2}\right) \wedge \eta\left(\left\{M\left(g^{n} x, g y, \frac{t}{2}\right), M\left(g y, g^{n} x, \frac{t}{2}\right)\right\}\right) \\
\geq & M\left(g y, g^{n} x, \frac{t}{2}\right) \wedge \eta\left(\left\{M\left(g^{n} x, g y, \frac{t}{2}\right)\right\}\right) .
\end{aligned}
$$

On taking limit as $n \rightarrow \infty$, we get that

$$
\begin{aligned}
M(g y, T y, t) & \geq M\left(y, y, \frac{t}{2}\right) \wedge \eta\left(M\left(y, y, \frac{t}{2}\right)\right) \\
& \geq 1 \wedge \eta\left(M\left(y, T y, \frac{t}{2}\right)\right) \geq M\left(y, y, \frac{t}{2}\right)=1 .
\end{aligned}
$$

Hence $g y \in T(y)$. Consequently, $y=g y \in T y$ and $y$ is a common fixed point of $T$ and $g$. If (b) holds, that is, for some $x \in C(g, T), g$ is $T$-commuting and $g^{2} x=g x$, then $g x=g^{2} x \in T(g x)$. Hence, $g x$ is a common fixed point of $T$ and $g$. If (c) holds, then, by the continuity of $g$ at $x$, we obtain that $x=g x \in T(x)$. Hence, $x$ is a common fixed point of $T$ and $g$.

Corollary 2.12 Let $(X, M, \wedge)$ be a complete fuzzy metric space, $T: X \rightarrow \mathcal{K}(X)$ and $g$ be a self-mapping on $X$ such that $T(X) \subseteq g(X)$. Suppose that there exists $r$ in $[0,1)$ such that for each $x, y \in X, t>0$ and $\eta \in \Omega$,

$$
M(g x, T x, t) \geq M(g x, g y, \varphi(r) t)
$$


implies

$$
H_{M}(T x, T y, r t) \geq \eta(M(g x, g y, t)) .
$$

Then $T$ and $g$ have a coincidence point provided that conditions (c-4) and (c-5) in Theorem 2.11 are satisfied. Moreover, $F$ and $g$ have a common fixed point if conditions (a)-(c) in Theorem 2.11 hold.

Now we prove the following corollary for a pair of single-valued self-mappings.

Corollary 2.13 Let $(X, M, \wedge)$ be a complete fuzzy metric space, $T, g: X \rightarrow X$ such that $T(X) \subseteq g(X)$. Suppose that there exists $r$ in $[0,1)$ such that for each $x, y \in X, t>0$ and $\eta \in \Omega$,

$$
M(g x, T x, t) \geq M(g x, g y, \varphi(r) t)
$$

implies

$$
M(T x, T y, r t) \geq \eta(M(g x, g y, t)) .
$$

Then $T$ and $g$ have a coincidence point provided that conditions (c-4) and (c-5) in Theorem 2.11 hold. Further, if $T$ and $g$ commute at $x$ for $x \in C(g, T)$, then $F$ and $g$ have a unique common fixed point.

Proof By Corollary 2.12, $C(g, T)$ is nonempty. Let $x \in C(g, T)$. Now, we claim that $g^{2} x=$ $\operatorname{Tgx}$. To prove the result, we need to show that $g x=g^{2} x$. If $g x \neq g^{2} x$, then $M(g x, T x, t)=1 \geq$ $M(g x, g y, \varphi(r) t)$. Note that

$$
\begin{aligned}
M\left(g x, g^{2} x, t\right) & >M\left(g x, g^{2} x, r t\right)=M(T x, \operatorname{Tg} x, r t) \\
& \geq \eta\left(M\left(g x, g^{2} x, t\right)\right)>M\left(g x, g^{2} x, t\right),
\end{aligned}
$$

a contradiction. Hence $g x=g^{2} x=T g x$ implies that $g x \in F(g, T)$. To prove the uniqueness, assume on the contrary that there exist $u$ and $w$ such that $u=g u=T u, w=g w=T w$ and $u \neq w$. Since $M(g u, T u, t)=1 \geq M(g u, g w, \varphi(r) t)$, by a given assumption we have

$$
\begin{aligned}
M(g u, g w, t) & =M(T u, T w, t) \geq M(T u, T w, r t) \\
& \geq \eta(M(g u, g w, t))>M(g u, g w, t),
\end{aligned}
$$

a contradiction. Hence $F(g, T)$ is singleton.

\section{Application in dynamic programming}

Now we present an application of our result in solving functional equations arising in dynamic programming.

Let $U$ and $V$ be Banach spaces, $W \subseteq U$ be a state space and $D \subseteq V$ be a decision space. A set of all feasible states or situations is the state space and a decision space is the resultant network formed by the nodes of feasible states and all the feasible decisions. It 
is desirable to find an optimal decision in the given state space using dynamic programming. Dynamic programming provides useful tools for mathematical optimization and computer programming as well. In particular, the problem of dynamic programming related to multistage process reduces to the problem of solving the functional equation

$$
\left\{\begin{aligned}
& p(x)=\sup _{y \in D}\{g(x, y)+\Phi(x, y, p(\tau(x, y)))\} \text { for } x \in W, \\
& q(x)=\sup _{y \in D}\{h(x, y)+\Psi(x, y, q(\tau(x, y)))\} \text { for } x \in W, \\
& \text { where } \tau: W \times D \rightarrow W, g, h: W \times D \rightarrow \mathbb{R} \text { and } \Phi, \Psi: W \times D \times \mathbb{R} \rightarrow \mathbb{R} .
\end{aligned}\right.
$$

However, for detailed background of the problem, we refer to [33-41].

Now, we study the existence and uniqueness of a bounded solution of the functional equation. For this we proceed as follows.

Let $B(W)$ be the set of all bounded real-valued functions on $W$. For $h, k \in B(W)$, define

$$
M(h, k, t)=e^{-\frac{d(h, k)}{t}},
$$

where

$$
d(h, k)=\sup _{x \in W}|h(x)-k(x)|=\|h-k\| .
$$

Then $(B(W), M, \cdot)$ is a complete fuzzy metric space. For every $(x, y) \in W \times D, h, k \in B(W)$ and $x \in W$, define

$$
\begin{aligned}
& \operatorname{Th}(x)=\sup _{y \in D}\{g(x, y)+\Phi(x, y, h(\tau(x, y)))\}, \\
& S h(x)=\sup _{y \in D}\{h(x, y)+\Psi(x, y, h(\tau(x, y)))\} .
\end{aligned}
$$

Suppose that the following conditions hold:

(D-1) $\Phi, \Psi, g$ and $h$ are bounded.

(D-2) There exist $r \in[0,1)$ and $\eta \in \Omega$ such that for every $(x, y) \in W \times D, h, k \in B(W)$ and $x \in W$, we have

$$
e^{-\left(\frac{d(\Phi(x, y, h(\tau(x, y))), \Phi(x, y, 1, k(\tau(x, y))))}{r t}\right)} \geq \eta\left(e^{-\left(\frac{d(S h(x), S k(x))}{t}\right)}\right) .
$$

(D-3) For any $h \in B(W)$ and $x \in W$, there exists $k \in B(W)$ such that $\operatorname{Th}(x)=S k(x)$.

(D-4) There exists $h \in B(W)$ such that $\operatorname{Th}(x)=\operatorname{Sh}(x)$ implies that $\operatorname{STh}(x)=\operatorname{TSh}(x)$.

Theorem 3.1 If conditions (D-1)-(D-4) are satisfied, then the system of functional equations (8) has a unique bounded and common solution in $B(W)$.

Proof Note that $T$ is a self-map on $B(W)$ and $(B(W), M, \cdot)$ is a complete fuzzy metric space. If $h, k \in B(W)$, then for every real number $\alpha$ and $x \in W$, there exist $y_{1}, y_{2} \in D$ such that

$$
\begin{aligned}
& T(h(x))<g\left(x, y_{1}\right)+\Phi\left(x, y_{1}, h\left(\tau_{1}\right)\right)+\alpha \quad \text { and } \\
& T(k(x))<g\left(x, y_{2}\right)+\Phi\left(x, y_{2}, k\left(\tau_{2}\right)\right)+\alpha,
\end{aligned}
$$

where $\tau_{1}=\tau\left(x, y_{1}\right)$ and $\tau_{2}=\tau\left(x, y_{2}\right)$. 
Then we have

$$
\begin{aligned}
& T(h(x)) \geq g\left(x, y_{2}\right)+\Phi\left(x, y_{1}, h\left(\tau_{1}\right)\right), \\
& T(k(x)) \geq g\left(x, y_{1}\right)+\Phi\left(x, y_{2}, k\left(\tau_{2}\right)\right) .
\end{aligned}
$$

Thus, from (9) and (12), we obtain

$$
\begin{aligned}
T(h(x))-T(k(x)) & <\Phi\left(x, y_{1}, h\left(\tau_{1}\right)\right)-\Phi\left(x, y_{2}, k\left(\tau_{2}\right)\right)+\alpha \\
& \leq\left|\Phi\left(x, y_{1}, h\left(\tau_{1}\right)\right)-\Phi\left(x, y_{2}, k\left(\tau_{2}\right)\right)\right|+\alpha .
\end{aligned}
$$

Also, from (10) and (11), we have

$$
\begin{aligned}
T(k(x))-T(h(x)) & <\Phi\left(x, y_{2}, k\left(\tau_{2}\right)\right)-\Phi\left(x, y_{1}, h\left(\tau_{1}\right)\right)+\alpha \\
& \leq\left|\Phi\left(x, y_{2}, k\left(\tau_{2}\right)\right)-\Phi\left(x, y_{1}, h\left(\tau_{1}\right)\right)\right|+\alpha .
\end{aligned}
$$

Since $\alpha>0$ was taken as an arbitrary number, we obtain

$$
\begin{aligned}
d(T(h(x)), T(k(x))) & =|T(h(x))-T(k(x))| \\
& \leq\left|\Phi\left(x, y_{2}, k\left(\tau_{2}\right)\right)-\Phi\left(x, y_{1}, h\left(\tau_{1}\right)\right)\right| .
\end{aligned}
$$

Thus

$$
\begin{aligned}
e^{-\frac{d(T(h(x)), T(k(x)))}{r t}} & \geq e^{-\frac{\left|\Phi\left(x_{y}, y_{2}, k\left(\tau_{2}\right)\right)-\Phi\left(x, y_{1}, h\left(\tau_{1}\right)\right)\right|}{r t}} \\
& \geq e^{-\left(\frac{d\left(\Phi\left(x, y_{2}, k\left(\tau_{2}\right)\right), \Phi\left(x, y_{1}, h\left(\tau_{1}\right)\right)\right)}{r t}\right)} \\
& \geq \eta\left(e^{-\frac{d(S h(x), S k(x))}{t}}\right) .
\end{aligned}
$$

Then it follows that

$$
M(T(h(x)), T(k(x)), r t) \geq \eta(M(\operatorname{Sh}(x), S k(x), t)) .
$$

Therefore all the conditions of Corollary 2.13 are satisfied for mappings $T$ and $S$. Hence the system of functional equations (8) has a unique bounded and common solution.

\section{Competing interests}

The authors declare that they have no competing interests.

\section{Authors' contributions}

All authors contributed equally to the writing of this paper. All authors read and approved the final manuscript.

\section{Author details}

'Department of Mathematics, National University of Computer and Emerging Sciences, Lahore, Pakistan. ${ }^{2}$ Department of Mathematics, School of Science and Technology, University of Management and Technology, C-II, Johar town, Lahore, Pakistan. ${ }^{3}$ Department of Mathematics and Applied Mathematics, University of Pretoria, Lynnwood road, Pretoria, 0002 , South Africa.

\section{Acknowledgements}

We appreciate the reviewer's careful reading and remarks which helped us to improve the paper.

Received: 26 November 2014 Accepted: 10 February 2015 Published online: 04 March 2015 


\section{References}

1. Banach, S: Sur les opérations dans les ensembles abstraits et leur application aux équations intégrales. Fundam. Math. 3, 133-181 (1922)

2. Kannan, R: Some results on fixed points - II. Am. Math. Mon. 76, 405-408 (1969)

3. Kikkawa, M, Suzuki, T: Some similarity between contractions and Kannan mappings. Fixed Point Theory Appl. 2008 Article ID 649749 (2008)

4. Suzuki, T: A generalized Banach contraction principle that characterizes metric completeness. Proc. Am. Math. Soc. 136, 1861-1869 (2008)

5. Damjanović, B, Đorić, D: Multivalued generalizations of the Kannan fixed point theorem. Filomat 25, 125-131 (2011)

6. Feng, Y, Liu, S: Fixed point theorems for multivalued contractive mappings and multivalued Caristi type mappings. J. Math. Anal. Appl. 317, 103-112 (2006)

7. Kikkawa, M, Suzuki, T: Three fixed point theorems for generalized contractions with constants in complete metric spaces. Nonlinear Anal. 69, 2942-2949 (2008)

8. Nadler, SB: Multivalued contraction mappings. Pac. J. Math. 30, 475-488 (1969)

9. Đorić, D, Lazović, R: Some Suzuki type fixed point theorems for generalized multivalued mappings and applications. Fixed Point Theory Appl. 2011, 40 (2011)

10. Zadeh, LA: Fuzzy sets. Inf. Control 8, 103-112 (1965)

11. Kramosil, I, Michalek, J: Fuzzy metric and statistical metric spaces. Kybernetika 11, 326-334 (1975)

12. George, A, Veeramani, P: On some results in fuzzy metric spaces. Fuzzy Sets Syst. 64, 395-399 (1994)

13. George, A, Veeramani, P: On some results of analysis for fuzzy metric spaces. Fuzzy Sets Syst. 90, 365-368 (1997)

14. Gregori, V, Morillaas, S, Sapena, A: Examples of fuzzy metrics and applications. Fuzzy Sets Syst. 170, 95-111 (2011)

15. Chauhan, S, Shatanawi, W, Kumar, S, Radenović, S: Existence and uniqueness of fixed points in modified intuitionistic fuzzy metric spaces. J. Nonlinear Sci. Appl. 7(1), 28-41 (2014)

16. Fang, JX: On fixed point theorems in fuzzy metric spaces. Fuzzy Sets Syst. 46, 107-113 (1992)

17. Grabiec, M: Fixed points in fuzzy metric spaces. Fuzzy Sets Syst. 27, 385-389 (1983)

18. Gregori, V, Romaguera, S: Some properties of fuzzy metric spaces. Fuzzy Sets Syst. 115, 485-489 (2000)

19. Gregori, V, Sapena, A: On fixed-point theorems in fuzzy metric spaces. Fuzzy Sets Syst. 125, 245-252 (2002)

20. Jain, S, Jain, S, Jain, LB: Compatibility of type (P) in modified intuitionistic fuzzy metric space. J. Nonlinear Sci. Appl. 3(2), 96-109 (2010)

21. Liu, Y, Li, Z: Coincidence point theorems in probabilistic and fuzzy metric spaces. Fuzzy Sets Syst. 158, 58-70 (2007)

22. Mihet, D: On the existence and the uniqueness of fixed points of Sehgal contractions. Fuzzy Sets Syst. 156, 135-141 (2005)

23. Mihet, D: On fuzzy contractive mappings in fuzzy metric spaces. Fuzzy Sets Syst. 158, 915-921 (2007)

24. Mihet, D: Common coupled fixed point theorems for contractive mappings in fuzzy metric spaces. J. Nonlinear Sci. Appl. 6(1), 35-40 (2013)

25. Ray, AD, Saha, PK: Fixed point theorems on generalized fuzzy metric spaces. Hacet. J. Math. Stat. 39, 1-9 (2010)

26. Razani, A: A contraction theorem in fuzzy metric space. Fixed Point Theory Appl. 2005(3), 257-265 (2005)

27. Som, T, Mukherjee, RN: Some fixed point theorems for fuzzy mappings. Fuzzy Sets Syst. 33, 213-219 (1989)

28. Rodríguez-López, J, Romaguera, S: The Hausdorff fuzzy metric on compact sets. Fuzzy Sets Syst. 147, $273-283$ (2004)

29. Schweizer, B, Sklar, A: Statistical metric spaces. Pac. J. Math. 10, 314-334 (1960)

30. Abbas, M, Ćirić, L, Damjanović, B, Khan, MA: Coupled coincidence and common fixed point theorems for hybrid pair of mappings. Fixed Point Theory Appl. (2012). doi:10.1186/1687-1812-2012-4

31. Haghi, RH, Rezapour, SH, Shahzad, N: Some fixed point generalizations are not real generalizations. Nonlinear Anal. 74, 1799-1803 (2011)

32. Hosseini, BH, Saadati, R, Amini, M: Alexandroff theorem in fuzzy metric spaces. Math. Sci. Res. J. 8(12), 357-361 (2004)

33. Baskaran, R, Subrahmanyam, PV: A note on the solution of a class of functional equations. Appl. Anal. 22(3-4), 235-241 (1986)

34. Bellman, R: Methods of Nonlinear Analysis. Volume II. Mathematics in Science and Engineering, vol. 61. Academic Press, New York (1973)

35. Bellman, R, Lee, ES: Functional equations in dynamic programming. Aequ. Math. 17, 1-18 (1978)

36. Bhakta, TC, Mitra, S: Some existence theorems for functional equations arising in dynamic programming. J. Math. Anal. Appl. 98, 348-362 (1984)

37. Liu, Z, Agarwal, RP, Kang, SM: On solvability of functional equations and system of functional equations arising in dynamic programming. J. Math. Anal. Appl. 297, 111-130 (2004)

38. Liu, Z, Wang, L, Kim, HK, Kang, SM: Common fixed point theorems for contractive type mappings and their applications in dynamic programming. Bull. Korean Math. Soc. 45(3), 573-585 (2008)

39. Pathak, HK, Cho, YJ, Kang, SM, Lee, BS: Fixed point theorems for compatible mappings of type (P) and applications to dynamic programming. Matematiche 50, 15-33 (1995)

40. Singh, SL, Mishra, SN: Coincidence theorems for certain classes of hybrid contractions. Fixed Point Theory Appl. 2010, Article ID 898109 (2010)

41. Singh, SL, Mishra, SN: On a Ljubomir Ćirić fixed point theorem for nonexpansive type maps with applications. Indian J. Pure Appl. Math. 33, 531-542 (2002) 